\title{
Targeting HIV Gag p24 to DICR on dendritic cells induces $T$ cell and potent and long-lasting antibody responses in non-human primates
}

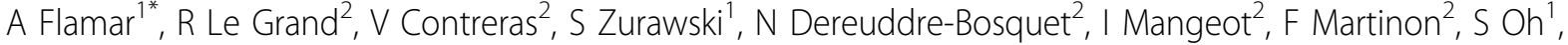 \\ J Banchereau', G Zurawski ${ }^{1}$, Y Levy ${ }^{3}$
}

From AIDS Vaccine 2012

Boston, MA, USA. 9-12 September 2012

\section{Background}

Targeting Dendritic Cells (DCs) with anti-DC receptor antibody-antigen fusion proteins is a novel approach in vaccine development for inducing potent humoral and cellular immune responses.

\section{Methods}

We engineered an anti-human DCIR recombinant antibody cross-reacting with the cynomolgus macaque receptor fused via the heavy chain C-terminus to HIV-1 Gagp24 protein (anti-DCIR.Gagp24). HIV patient PBMC cultures were incubated with anti-DCIR and control hIgG4.Gagp24 fusion proteins. After 10 days, the total T cells were challenged with HIV Gagp24 peptide pools, and then antigen-specific cytokine production was detected using intracellular staining. Macaques were also immunized i.d. 3 times with anti-DCIR.Gagp24 or control hIgG4.Gagp24 with or without polyI:C. Gagp24-specific IgG titers from serum were measured by ELISA and the magnitude of the antigen-specific IFN $\gamma$ responses was assessed by ELISPOT.

\section{Results}

In vitro, low doses of anti-DCIR Gagp24 prototype vaccine, but not the control hIgG4.Gagp24, expand of Gagp24-specific $\mathrm{T}$ cells. These in vitro-expanded antigen-specific $\mathrm{T}$ cells were multifunctional, simultaneously producing multiple cytokines (IFN $\gamma$, TNF $\alpha$ and MIP-1 $\beta$ ). In vivo, in non-adjuvanted naïve animals, serum antiGagp24 antibodies were detectable 2 weeks after priming and titers were substantially increased after the 1 st and the 2nd boost with anti-DCIR.Gagp24 and were durable, while in the control hIgG4.Gagp24 group the responses were minimal. Poly I:C increased the magnitude of the responses in the anti-DCIR.Gagp24 and hIgG4.Gagp24 groups to a similar level in both groups. $\mathrm{T}$ cell responses induced by anti-DCIR Gagp24 could be enhanced after priming with a recombinant modified vaccinia virus Ankara (MVA) encoding HIV Gag/Pol/Nef. Boosting with anti-DCIR.Gagp24 plus poly I:C generated high titers of anti-Gagp24 antibody titers and strongly enhanced IFN $\gamma$-producing $\mathrm{T}$ cells following priming with MVA HIV Gag/Pol/Nef.

\section{Conclusion}

Our results demonstrate that heterologous prime-boost immunization with vectors and DC-targeting proteinbased vaccines is a promising vaccination approach to optimize humoral and cellular immunity for therapeutic and preventive applications against AIDS.

\section{Author details}

'Baylor Institute for Immunology Research, Dallas, TX, USA. ${ }^{2}$ CEA, Fontenayaux-Roses, France. ${ }^{3}$ INSERM U955, Faculté de Médecine de Créteil, Creteil, France.

Published: 13 September 2012

doi:10.1186/1742-4690-9-S2-P358

Cite this article as: Flamar et al: Targeting HIV Gag p24 to DICR on dendritic cells induces $T$ cell and potent and long-lasting antibody responses in non-human primates. Retrovirology 2012 9(Suppl 2):P358.

'Baylor Institute for Immunology Research, Dallas, TX, USA

Full list of author information is available at the end of the article

C 2012 Flamar et al; licensee BioMed Central Ltd. This is an Open Access article distributed under the terms of the Creative Commons 